\title{
A NOTE ON THE OPTIMAL REPLACEMENT PROBLEM
}

\author{
LAM YEH, ${ }^{*}$ The Chinese University of Hong Kong
}

\begin{abstract}
In this note, we study a new repair replacement model for a deteriorating system, in which the successive survival times of the system form a geometric process and are stochastically non-increasing, whereas the consecutive repair times after failure also constitute a geometric process but are stochastically non-decreasing. Two kinds of replacement policy are considered, one based on the working age of the system and the other one determined by the number of failures. The explicit expressions of the long-run average costs per unit time under these two kinds of policy are calculated.
\end{abstract}

REPLACEMENT PROBLEM; STOCHASTIC MONOTONICITY; RENEWAL PROCESSES

\section{Introduction}

Most repair replacement models assume that a failure system after repair will yield a functioning system which is 'as good as new' i.e., the system is renewed after each failure. An alternative assumption is to assume that a failure system after repair will function again, but has the same failure rate and the same effective age at the time of failure: this leads to the minimal repair model. In both cases, the repair times are often negligible (see e.g. Barlow and Proschan (1965) or Ascher and Feingold (1984)), although there exist a large number of repair replacement models where the repair times are taken into account (see e.g. Morimura (1970)).

However, for deteriorating systems, the problem is different from that described above. For example, in machine maintenance problems, after repair, because of the deterioration, the functioning time of a machine will become shorter and shorter so the total functioning time or the total life of the machine must be finite. On the other hand, in view of the ageing and accumulative wear, the repair time will become longer and longer and tend to infinity: i.e., finally the machine is non-repairable. Therefore, we have to consider a repair replacement model for deteriorating systems as follows: the successive survival times are non-increasing and will eventually die out, so that the total life of the system is finite; moreover, the consecutive repair times are non-decreasing and will finally tend to infinity.

One possible way of modelling these deteriorating systems is to use the non-homogeneous Poisson process. Another possible way is to introduce the following geometric process.

Definition 1. Given a sequence of random variables $X_{1}, X_{2}, \cdots$ if for some $a>0$, $\left\{a^{n-1} X_{n}, n=1,2, \cdots\right\}$ forms a renewal process, then $\left\{X_{n}, n=1,2, \cdots\right\}$ is a geometric process. $a$ is called the parameter of the geometric process.

Definition 2. A geometric process is called a non-increasing geometric process if $a \geqq 1$ and a non-decreasing geometric process if $a \leqq 1$.

Note, if $a=1$, then the geometric process reduces to a renewal process.

It is easy to show that for a geometric process, if $a>1$, then it is stochastically decreasing and converges to 0 in probability; furthermore, if $a>1$, then $U=\sum_{n=1}^{\infty} X_{n}$ converges in the

Received 18 June 1986; revision received 25 January 1988.

* Postal Address: Department of Statistics, The Chinese University of Hong Kong, Shatin, N.T., Hong Kong. 
sense of mean square with $P(U<\infty)=1$. Whereas, if $a<1$, then it is stochastically increasing and tends to infinity in probability (see Lam Yeh (1987) for more details). Therefore, for the deteriorating systems considered above, if its successive survival times are increasing geometrically with a constant rate, then we can model them by using a non-increasing geometric process. Otherwise, if its successive survival times are decreasing in a general way, we can still use a non-increasing geometric process as an approximation for modelling the successive survival times; this is exactly like using a linear function as an approximation of a general function. Similarly, we can use a non-decreasing geometric process for formulating its consecutive repair times either precisely or approximately.

\section{Model}

Now, we are able to state the new repair replacement model as below.

Assumption 1. At the beginning, a new system is used.

Assumption 2. Whenever the system fails, we can repair it. Let $X_{n}$ be the survival time after the $(n-1)$ th repair, then $\left\{X_{n}, n=1,2, \cdots\right\}$ forms a non-increasing geometric process with parameter $a \geqq 1$ and $E\left(X_{1}\right)=\lambda>0$.

Assumption 3. Let $Y_{n}$ be the repair time after the $n$th failure, then $\left\{Y_{n}, n=1,2, \cdots\right\}$ constitutes a non-decreasing geometric process with parameter $0<b \leqq 1$ and $E\left(Y_{1}\right)=\mu \geqq 0$, $\mu=0$ means that the repair time is negligible.

Assumption 4. $\left\{X_{n}, n=1,2, \cdots\right\}$ and $\left\{Y_{n}, n=1,2, \cdots\right\}$ are independent.

Assumption 5. The repair cost rate is $C$, the reward rate whenever the system is working is $r$, without loss of generality, we assume that $r=1$.

Two kinds of replacement policy are considered in this model.

(i) A replacement policy $T$ is a policy of which we replace the system whenever the working age of the system reaches $T$.

The working age $T$ of a system at time $t$ is the cumulative survival time by time $t$, i.e.,

$$
T= \begin{cases}t-V_{n}, & U_{n}+V_{n} \leqq t<U_{n+1}+V_{n} \\ U_{n+1}, & U_{n+1}+V_{n} \leqq t<U_{n+1}+V_{n+1}\end{cases}
$$

where

$$
U_{n}=\sum_{i=1}^{n} X_{i}, \quad V_{n}=\sum_{i=1}^{n} Y_{i} \quad \text { and } \quad U_{0}=0, \quad V_{0}=0 .
$$

(ii) A replacement policy $N$ is a policy of which we replace the system at the time of $N$ th failure since the last replacement.

Assumption 6. The system may be replaced sometime by a new and identical one. The replacement cost under policy $T$ is $C_{1}$; the replacement cost under policy $N$ is $C_{2}$.

Under replacement policy $T$ or $N$, the problem is to determine an optimal replacement policy $T^{*}$ or $N^{*}$ respectively, such that the long-run average cost per unit time is minimized.

\section{Long-run average cost}

Let $T_{1}$ be the first replacement time, in general, for $n \geqq 2$, let $T_{n}$ be the time between $(n-1)$ th replacement and $n$th replacement, then obviously, $\left\{T_{n}, n=1,2, \cdots\right\}$ forms a renewal process. It is well known that the long-run average cost per unit time is given by

$$
\frac{\text { the expected cost incurred in a cycle }}{\text { the expected length of a cycle }}
$$

where a cycle is the time between two consecutive replacements (see, e.g. Ross (1970)). 
Under the replacement policy $T$, denote the length of a cycle by $W$, then

$$
W=T+V_{K}, \quad U_{K}<T \leqq U_{K+1}, \quad K=0,1, \cdots .
$$

From Definition 1 and Assumption 3, it follows that $E\left(Y_{k}\right)=\mu / b^{k-1}$. Then

$$
\begin{aligned}
E(W) & =E\left(T+\sum_{k=1}^{K} Y_{k}\right) \\
& =E\left[T+\sum_{k=1}^{\infty} Y_{k} I\left(U_{k}<T\right)\right] \\
& =T+\mu \sum_{k=1}^{\infty} \frac{1}{b^{k-1}} F_{k}(T-0)
\end{aligned}
$$

where $I$ is the indicator and $F_{k}$ is the distribution of $U_{k}$.

Thus, from (2) the long-run average cost per unit time $C_{1}(T)$ under policy $T$ is given by

where

$$
\begin{aligned}
C_{1}(T) & =\frac{C \mu \sum_{k=1}^{\infty} \frac{1}{b^{k-1}} F_{k}(T-0)+C_{1}-T}{T+\mu \sum_{k=1}^{\infty} \frac{1}{b^{k-1}} F_{k}(T-0)} \\
& =C_{1}^{*}(T)-1,
\end{aligned}
$$

$$
C_{1}^{*}(T)=\frac{(C+1) \mu \sum_{k=1}^{\infty} \frac{1}{b^{k-1}} F_{k}(T-0)+C_{1}}{T+\mu \sum_{k=1}^{\infty} \frac{1}{b^{k-1}} F_{k}(T-0)} .
$$

Similarly, under replacement policy $N$, the long-run average cost per unit time $C_{2}(N)$ is given by

$$
\begin{aligned}
C_{2}(N) & =\frac{C \mu \sum_{k=1}^{N-1} \frac{1}{b^{k-1}}+C_{2}-\lambda \sum_{k=1}^{N} \frac{1}{a^{k-1}}}{\lambda \sum_{k=1}^{N} \frac{1}{a^{k-1}}+\mu \sum_{k=1}^{N-1} \frac{1}{b^{k-1}}} \\
& =C_{2}^{*}(N)-1
\end{aligned}
$$

where

$$
C_{2}^{*}(N)=\frac{(C+1) \mu \sum_{k=1}^{N-1} \frac{1}{b^{k-1}}+C_{2}}{\lambda \sum_{k=1}^{N} \frac{1}{a^{k-1}}+\mu \sum_{k=1}^{N-1} \frac{1}{b^{k-1}}}, \quad N=1,2, \cdots
$$

Finally, we can determine the optimal replacement policy $T^{*}$ and $N^{*}$ by minimizing $C_{1}(T)$ (or $C_{1}^{*}(T)$ ) and $C_{2}(N)$ (or $C_{2}^{*}(N)$ ) respectively. Furthermore, the minimization procedure can be done by analytical or numerical methods.

In practice, we prefer to adopt the optimal policy $N^{*}$ rather than use the optimal policy $T^{*}$, because of the much simpler form of (6) or (7).

\section{Acknowledgement}

The author is grateful to the referee for his valuable comments which made much improvement in the presentation. 


\section{References}

AsCher, H. AND Feingold, H. (1984) Repairable Systems Reliability. Marcel Dekker, New York. Barlow, R. E. AND Proschan, F. (1965) Mathematical Theory of Reliability. Wiley, New York. LAM YeH (1987) Geometric Processes and Replacement Problem. Technical Report 42, Department of Statistics, The Chinese University of Hong Kong.

MORIMURA, H. (1970) On some preventive maintenance policies for IFR. J. Operat. Res. Soc. Japan. 12, 94-124.

Ross, S. M. (1970). Applied Probability Models with Optimization Applications. Holden-Day, San Francisco.

Ross, S. M. (1983) Stochastic Processes. Wiley, New York. 